\title{
Evolução tardia da operação de Cox para fibrilação atrial em valvopatia mitral
}

\author{
Marcelo B. JATENE* ${ }^{\star}$, Eduardo SOSA ${ }^{\star *}$, Fábio B. JATENE* ${ }^{\star}$, Flávio TARASOUTCHI ${ }^{\star *}$, Ana Cristina \\ MONTEIRO**, Pedro R. SALERNO***, Luiz Carlos Bento de SOUZA***, José Carlos PACHÓN \\ MATEOS $^{\star \star *}$, Adib D. JATENE*
}

RBCCV $44205-254$

\begin{abstract}
JATENE\$M. B.; SOSA, E.; JATENE, F. B.; TARASOUTCHI, F.; MONTEIRO, A. C.; SALERNO, P. R.; SOUZA, L. C. B.; PACHÓN MATEOS, J. C.; JATENE, A. D. - Evoluçāo tardia da operação de Cox para fibrilação atrial em valvopatia mitral. Rev. Bras. Cir. Cardiovasc., 10 (1): 18-24, 1995.
\end{abstract}

\begin{abstract}
RESUMO: A operação de Cox para fibrilação atrial (FA), descrita inicialmente para os casos de FA crônica e ou paroxística, vem tendo, em nosso meio, emprego freqũente em pacientes (pts) valvopatas mitrais portadores de FA, com controle da arritmia a médio e longo prazo. Entre julho 91 e outubro 94, 32 pts (25 femininos) portadores de FA crônica e valvopatia mitral foram operados. A idade média foi de 50,2 anos ( 35 a 68 ), sendo a valvopatia de origem reumática em 30 pts e mixomatosa em $2 ; 81,2 \%$ dos pacientes encontravam-se em CF IV (NYHA), sendo que 9 haviam sido previamente operados e 4 tiveram episódio prévio de embolia sistêmica. O diâmetro médio do AE era de $5,5 \mathrm{~cm}$ e a PSAP de $55 \mathrm{mmHg}$. Todos os pts foram operados por esternotomia mediana, seguindo a técnica original descrita por Cox (CoxI) em 18 pts e a técnica modificada (Cox III) em 14 pts. Crioablação dos anéis mitral, tricúspide e seio coronário foi utilizada em 25 pts. Em 17 pts a valva mitral foi substituida por bioprótese de $\mathrm{PB}$ e conservada em 15 pts. Foram observados 2 óbitos hospitalares (baixo débito e insuficiência hepática); 14 pts fizeram uso de MP temporário no pósoperatório imediato e 4 apresentaram insuficiência respiratória com boa evoluçăo. Um paciente apresentou morte súbita domiciliar, uma semana após a alta. Em 25 pts com evoluçäo superior a 6 meses (média 25 m), 24 encontram-se em rítmo regular (sinusal em 19 e juncional em 5), assintomáticos e com FC média de 71,5 bpm. Contração atrial foi demonstrada pelo ECO, sendo menos efetiva no AE. Um paciente foi submetido a implante de MP definitivo por bradicardia sinusal no 2 mês de PO. Em conclusão, a operação de Cox vem mostrando resultados reprodutíveis na FA associada à doença mitral, com obtenção de rítmo regular e contração atrial presente. Não houve recorrência de FA no período avaliado.
\end{abstract}

DESCRITORES: Operação de Cox, evolução tardia. Fibrilação atrial, cirurgia.

\section{INTRODUÇÃO}

A fibrilação atrial (FA) é arritmia cardíaca comum na população geral, variando de $0,15 \%$ a $1 \%$, sendo que em pacientes (pts) com idade superior a 60 anos chega a atingir de 8 a $17 \% 2,3,15,19,23$ $25,28,29$. A ocorrência de FA em portadores de valvopatia mitral é freqüente, em especial de etiologia reumática, mais comumente nos casos de estenose ou dupla lesão mitral.

Dentre os problemas relacionados à presença da FA, três assumem maior importância: 1) rítmo irregular com freqüência cardíaca elevada; 2) dimi-

\footnotetext{
Trabalho realizado no Instituto do Coração do Hospital das Clinicas da Faculdade de Medicina da Universidade de São Paulo e no Hospital do Coração da Associação do Sanatório Sírio, Sāo Paulo, SP, Brasil.

Apresentado ao $22^{\circ}$ Congresso Nacional de Cirurgia Cardiaca. Brasília, DF, 30 de março a 1 de abril, 1995.

* Do Instituto do Coração da USP e do Hospital do Coração da ASS.

${ }^{\star *}$ Do Instituto do Coração da USP.

*** Do Hospital do Coração da ASS.

Endereço para correspondência: Marcelo Jatene, Av. Dr. Enéas de Carvalho Aguiar, 44. Divisão Cirúrgica. CEP 05403-000 São Paulo, SP, Brasil. Tel. (011) 898.5432. Fax: (011) 282.2354
} 
JATENE, M. B.; SOSA, E.; JATENE, F. B.; TARASOUTCHI, F.; MONTEIRO, A. C.; SALERNO, P. R.; SOUZA, L. C. B.; PACHÓN MATEOS, J. C.; JATENE, A. D. - Evolução tardia da operação de Cox para fibrilação atrial em valvopatia mitral. Rev. Bras. Cir. Cardiovasc., 10 (1): 18-24, 1995.

nuição do tempo de enchimento ventricular, com conseqüente distúrbio hemodinâmico e provável regurgitação valvar; 3 ) ocorrência de trombose atrial com risco elevado de fenômenos tromboembólicos.

O controle medicamentoso ou por desfibrilação elétrica da FA é possível, porém sua manutenção a médio e longo prazo em rítmo sinusal é geralmente difícil,com recorrências freqũentes em grande número de pts, às vezes de forma irreversível. Outro inconveniente da FA é a possibilidade do aparecimento de episódios paroxísticos, que, na maior parte das vezes, requerem atendimento emergencial. O controle da freqüência cardíaca a níveis aceitáveis em presença de FA não diminui o risco de episódios trombo-embólicos. Segundo FISHER ${ }^{16}$, cerca de $33 \%$ de todos os episódios de trombo-embolismo estão relacionados com a presença de FA e, destes, $60 \%$ podem levar a seqüelas definitivas ou morte.

Por causa da dificuldade em se obter e manter o rítmo sinusal, vários procedimentos cirúrgicos ou por cateterismo intervencionista foram propostos nos últimos 10 anos $18,26,27,30$, sendo que, em 1991, Cox descreveu uma técnica cirúrgica (Cox I) 6, 12 que procurava de uma forma definitiva: 1) interromper todos os circuitos de reentrada; 2) restabelecer o controle atrial dos batimentos cardíacos; 3 ) permitir que o estímulo atrial despolarize e ative os ventrículos de uma forma regular. Dessa forma, os inconvenientes da FA poderiam ser definitivamente controlados. Com o acúmulo da experiência, problemas relacionados com baixa resposta cronotrópica foram observados, levando COX et alii ${ }^{4,13}$ a descreverem modificações na técnica original (Cox III).

Nossa experiência com as duas técnicas descritas por Cox no tratamento da FA em valvopatia mitral, bem como seus resultados tardios seräo abordados.

\section{CASUÍSTICA E MÉTODOS}

No período entre julho 91 e outubro 94,34 pts com idade média de 50,2 anos (35 a 68 anos) portadores de FA crônica e valvopatia mitral foram operados. Vinte e seis $(76,4 \%)$ pts eram do sexo feminino e todos apresentavam FA há pelo menos 1 ano, a despeito de terapêutica medicamentosa. A valvopatia mitral era de origem reumática em 31 $(91,1 \%)$ pts e mixomatosa em $3(8,9 \%)$; em 2 pts havia presença de insuficiência tricúspide importante associada; 1 paciente apresentava estenose aórtica e outro paciente era portador de doença coronária uniarterial (lesão de $75 \%$ no ramo interventricular anterior). Os diferentes diagnósticos das lesões valvares estão apresentados na Tabela I.
TABELA 1

DIAGNÓSTICO E LESÕES ASSOCIADAS

\begin{tabular}{lr}
\hline EMi isolada & 18 \\
DLMi & 8 \\
IMi & 3 \\
EMi + ITric & 2 \\
EMi + EAo & 1 \\
Disfunção de prótese mitral & 1 \\
DLMi + ICo & 1 \\
\hline
\end{tabular}

$\mathrm{EMi}=$ Estenose Mitral; DLMi $=$ Dupla Lesão Mitral; IMi = Insuficiência Mitral; EAO = Estenose Aórtica; $\mid$ Tric = Insuficiência Tricúspide; $1 \mathrm{Co}=$ Insuficiência Coronariana

Do ponto de vista clínico, os sintomas eram em sua quase totalidade relacionados à doença valvar mitral, sendo esta a causa principal para a indicação cirúrgica. Vinte e oito $(82,3 \%)$ pts encontravamse em CF IV (NYHA), com episódio prévio de edema agudo de pulmão em 2 pts, e $6(17,6 \%)$ estavam em CF III. Nove $(26,4 \%)$ pts haviam sido previamente operados, com realização de 6 comissurotomias e 3 trocas valvares mitrais, e 1 paciente havia sido submetido a valvoplastia mitral com cateter balão; destes, 7 pts evoluíram com reestenose mitral e $3 \mathrm{com}$ disfunção de prótese biológica ( 1 caso de endocardite bacteriana); em 4 pts ocorreram episódios trombo-embólicos prévios à operação, com evolução favorável sem seqũelas em 3 e aparecimento de disartria em 1 paciente.

O diagnóstico de valvopatia mitral foi feito clinicamente e confirmado por ecocardiograma (Eco) e cateterismo cardíaco em todos os pts. Dentre os parâmetros medidos pelo Eco, observou-se importante aumento de átrio esquerdo, que variou de 4,4 a $6,5 \mathrm{~cm}(5,5 \mathrm{~cm})$; a pressão sistólica de artéria pulmonar variou de 37 a $70 \mathrm{mmHg}(55,1 \mathrm{mmHg})$; trombose atrial esquerda foi observada em 4 pts.

\section{TÉCNICA CIRÚRGICA}

Todos os pts foram operados por esternotomia mediana com o uso de circulação extracorpórea (CEC). A proteção miocárdica em todos os pts constou do emprego de hipotermia moderada $\left(25^{\circ} \mathrm{C}\right)$, hipotermia tópica com soro gelado no pericárdio e indução de parada cardíaca com solução cardioplégica cristalóide de St. Thomas, infundida na aorta ascendente com gelco 14 ou diretamente nos óstios coronarianos quando da presença de algum grau de regurgitação aórtica (em 4 pts); a cardioplegia era repetida da forma descrita, a cada 25 a 30 minutos.

Após abertura mediana do pericárdio e lise de aderências nos casos de reoperações, o primeiro passo da cirurgia consistiu de dissecção extensa e 
JATENE, M. B.; SOSA, E.; JATENE, F. B.; TARASOUTCHI, F.; MONTEIRO, A. C.; SALERNO, P. R.; SOUZA, L. C. B.; PACHÓN MATEOS, J. C.; JATENE, A. D. - Evoluçāo tardia da operaçāo de Cox para fibrilaçāo atrial em valvopatia mitral. Rev. Bras. Cir. Cardiovasc., $10(1): 18-24,1995$.

cuidadosa de todo o coração, liberando as veias cava superior e inferior, veias pulmonares, aorta e tronco pulmonar de todos os tecidos extracardiacos, reflexões pericárdicas e sulcos anatômicos. Em seguida procedeu-se à heparinização $(4 \mathrm{mg} / \mathrm{kg}$ de peso) e canulação das duas cavas com introdução de cânulas de $1 / 4^{\prime \prime}$ e $3 / 8^{\prime \prime}$, respectivamente, nas veia cava superior e inferior, o mais distal possível; a canulação da aorta ascendente foi feita de maneira habitual.

Realizou-se a técnica de Cox I nos primeiros 21 pts e a técnica de Cox III nos outros 13 pts. As aurículas direita e esquerda foram ressecadas em todos os pts, tendo sido identificada e removida trombose atrial esquerda em 8 pts. Crioablação dos anéis mitral e tricúspide e do seio coronário foi realizada em 27 pts, segundo a técnica descrita por Cox; nos outros 7 pts realizou-se apenas dissecção cuidadosa dos anéis mitral e tricúspide e seio coronário, sem aplicação de criotermia. Em todos os pts, a valva mitral foi abordada inicialmente, procedendo-se ao seu tratamento antes da realização da operação de Cox; nos casos operados com a técnica de Cox I, o acesso à valva mitral foi feito por incisão no topo do átrio esquerdo e septo interatrial, com boa exposição da valva; nos pts operados com a técnica de Cox III, a valva mitral foi abordada por incisão habitual no átrio esquerdo.

Em $17(50 \%)$ pts a valva mitral foi substituída por bioprótese de pericárdio bovino e conservada em $17(50 \%)$ pts, com 14 comissurotomias e 3 plásticas valvares. Os demais procedimentos cirúrgicos realizados estão expostos na Tabela 2 .

O tempo de CEC variou de 115 a $215(156,3)$ minutos, com tempo médio de pinçamento de aorta de 121,8 (50 a 174) minutos. A solução cardioplégica infundida era toda aspirada para fora do circuito de CEC, através de aspirador externo posicionado no seio coronário, dentro do átrio direito.

\section{RESULTADOS}

A mortalidade hospitalar foi de 5,8\% (2 pts); em um dos pts, o baixo débito cardíaco foi a causa de óbito, independente do uso de drogas inotrópicas e balão intra-aórtico; no outro paciente (comissurotomia mitral prévia há 12 anos), identificou-se severa disfunção hepática. Em ambos os pts obtevese ritmo regular no período pós operatório correspondente.

O primeiro paciente da série foi reoperado para revisão de hemostasia, com boa evolução. Em 4 pts, suporte ventilatório por mais de 48 hs foi necessário, com evolução favorável; baixo débito cardíaco, com necessidade de suporte inotrópico foi
TABELA 2 PROCEDIMENTOS CIRÚRGICOS

TVMi

Comiss $\mathrm{Mi}$

Plastia Mi

Comiss $\mathrm{Mi}+$ Comiss $\mathrm{Ao}$

Comiss $\mathrm{Mi}+$ De Vega Tric

$\mathrm{TVMi}+$ Rev Mioc

16
12
3
1
1
1

TVMi = Troca Valva Mitral; ComissMi = Comissurotomia Mitral; ComissAo = Comissurotomia Aórtica; $\mathrm{De}$ Vega Tric $=\mathrm{De}$ Vega Valva Tricúspide; RevMioc $=$ Revascularizaçăo do Miocárdio

observado em 3 pts; 14 (41,1\%) pts fizeram uso de marcapasso (MP) temporário no pós-operatório imediato, devido a bradicardia sinusal, com desconexão antes da alta hospitalar; arritmias atriais foram observadas em $6(17,6 \%)$ pts, sendo que 4 apresentaram FA, revertida e controlada com tratamento medicamentoso ou ajuste metabólico.

Um paciente foi reoperado no $24^{\circ} \mathrm{PO}$, para substituição de bioprótese, infectada por Enteroccocus fecalis, com boa evolução e manutenção de ritmo regular durante todo o procedimento.

Outro paciente apresentou infecção pulmonar severa no $2^{\circ}$ mês de $\mathrm{PO}$, com óbito no $48^{\circ} \mathrm{PO}$ devido a complicações infecciosas; o rítmo manteve-se sinusal. Mais recentemente, uma paciente apresentou morte súbita domiciliar, uma semana após a alta hospitalar; esta paciente havia sido submetida a troca valvar (comissurotomia mitral há 9 anos) e operação de Cox III, com boa evolução e alta hospitalar no $12^{\circ} \mathrm{PO}$, em ritmo sinusal (FC 60-65 bpm).

\section{EVOLUÇÃO TARDIA}

Em 30 pts com evolução superior a 6 meses $(6$ a 45 meses), 27 (90\%) pts se mantêm em ritmo atrial regular, sendo sinusal em 23 e juncional em 3 pts. A FC média observada foi de $71,5 \mathrm{bpm}$. Um paciente que se encontrava em ritmo juncional bradicárdico (45 a $50 \mathrm{bpm}$ ) foi submetido a implante de MP definitivo 3 meses após a operação (portador de disfunção de bioprótese mitral por endocardite bacteriana); o ritmo irregular foi observado em 3 pts, que desde o início do seguimento apresentaram FA, com difícil controle da arritmia, mesmo recebendo medicações antiarrítmicas; 2 destes pts foram submetidos à operação de Cox $I$ e 1 foi operado com a técnica de Cox III. Durante o período avaliado, nenhum dos pts, que, após seis meses de evolução estavam em ritmo regular, apresentou recorrência da FA. 
JATENE, M. B.; SOSA, E.; JATENE, F. B.; TARASOUTCHI, F.; MONTEIRO, A. C.; SALERNO, P. R.; SOUZA, L. C. B.; PACHÓN MATEOS, J. C.; JATENE, A. D. - Evolução tardia da operação de Cox para fibrilação atrial em valvopatia mitral. Rev. Bras. Cir. Cardiovasc., 10 (1): 18-24, 1995.

No mesmo período, 15 pts tiveram seu rítmo cardíaco avaliado por Holter de $24 \mathrm{hs}$, após infusão de atropina endovenosa e após teste de esforço (TE), segundo o protocolo de Ellestad; a contração atrial foi estudada por Eco. Avaliando-se os resultados do Holter, observou-se a ocorrência de taquicardia atrial não sustentada assintomática em $63 \%$ dos pts ( 3 a 45 batimentos/hora), sem registro de FA. Após infusão de atropina $(0,04 \mathrm{mg} / \mathrm{kg}$ de peso), a FC pré e pós atropina foi de $73,6+/-10,5$ e $74,8+/-10,6 \mathrm{bpm}$, respectivamente $(\mathrm{p}=0,264)$; após TE, a FC pré e pós foi de $72,8+/-11,3$ e $105+/-16,7$, respectivamente $(p=0,00021)$.

Contração atrial eficiente foi demonstrada pelo Eco em todos os pts estudados, observando-se diminuição da onda $A$ no átrio esquerdo quando comparado com 0 átrio direito. Constatou-se também a diminuição do diâmetro médio do átrio esquerdo para $4,7 \mathrm{~cm}(3,8$ a $5,5 \mathrm{~cm})$, com fração de ejeção normal.

Sob o ponto de vista clínico, $25(83,3 \%)$ pts encontram-se assintomáticos, sem uso de drogas antiarrítmicas, $3(10 \%)$ pts em ICC CF II (NYHA), porém sem progressão dos sintomas, com regurgitação mitral discreta a moderada. Os 3 pts em rítmo de FA vêm fazendo uso apenas de digitálicos, com freqüência cardíaca controlada, entre 70 e $80 \mathrm{bpm}$.

\section{COMENTÁRIOS}

Embora a operação de $\operatorname{Cox} 7,8$ tenha sido originalmente descrita para casos de FA crônica ou paroxística, sua aplicação em pts portadores de valvopatias, predominantemente em valva mitral, também pode ser opção importante. Em função da ocorrência freqüente, em nosso meio, da valvopatia reumática, decidimos iniciar a experiência com a operação de Cox nos pts valvopatas mitrais portadores de $F A$, com indicação para operar a valva mitral e, simultaneamente, realizar o tratamento da FA. Em nossos primeiros 10 pts operados, 9 eram valvopatas mitrais e o resultado obtido por JATENE et alii 20 foi satisfatório. Complementando essa experiência inicial, pudemos comprovar a eficácia da operação de Cox em controlar a FA, com manutenção dos resultados a longo prazo. Resultados também satisfatórios no controle da FA em valvopatas foram obtidos em nosso meio por GREGORI et alii ${ }^{17}$ e por McCARTHY et alii ${ }^{22} \mathrm{e}$ COX et alii ${ }^{7}$ em um número pequeno de pts; KOSAKAI et alii ${ }^{21}$, em uma série de 62 pts, todos valvopatas mitrais, obtiveram sucesso no controle da FA em $84 \%$ deles.

Alguns aspectos do emprego da operação de Cox em pts valvopatas devem ser considerados, como por exemplo o fato de que geralmente apresentam dimensões aumentadas das câmaras cardíacas, em especial o átrio esquerdo; nestas condições, o emprego da operação de Cox em pts com grandes átrios poderia deixar faixas de tecido atrial suficientemente largas para favorecer o fenômeno de reentrada e desencadear a FA; KOSAKAI et alii 21 atribuíram ao tamanho aumentado do $A E$ o insucesso registrado em cerca de $10 \%$ de seus pts. Em nossa casuística, onde o tamanho médio do $\mathrm{AE}$ foi de 5,5 $\mathrm{cm}$, conseguiu-se o controle da FA em $90 \%$ dos pts; no entanto, em 2 pts que permaneceram em FA a longo prazo, o AE media cerca de $6,0 \mathrm{~cm}$, ○ que talvez tenha sido um dos principais motivos para o insucesso da operação. No sentido de evitar que o tamanho exagerado do $A E$ possa interferir no resultado da cirurgia, algumas medidas técnicas passaram a ser tomadas, como a ressecção de faixas de tecido atrial, além de suturas que englobem mais tecido entre os pontos, visando diminuir os átrios e tentar desta forma evitar a reentrada.

Outro aspecto a ser analisado é o dos pts com operações anteriores, com incisões atriais prévias; COX et alii ${ }^{7}$ relatam um maior índice de morbidade em pts já previamente operados, além de considerar que átrios com cicatrizes prévias não têm a mesma capacidade de conduzir o estímulo através de suas paredes. Em nossa experiência, algumas das complicações observadas ocorreram em pts com cirurgias anteriores; dos 4 pts que evoluíram para óbito, 2 haviam sido operados (comissurotomia mitral) e dos 3 pts que ainda persistem em FA, 2 haviam sido submetidos a procedimentos na valva mitral (comissurotomia mitral e valvoplastia com cateter balão); a única paciente que necessitou implante de MP definitivo havia sido operada há 7 anos (troca valvar mitral). Desta forma, a utilização da operação de Cox em reoperações parece estar relacionada com aumento da morbi mortalidade, nos dando a impressão de que seu uso deve ser limitado a casos especiais, sem que faça parte dos procedimentos de rotina.

Do ponto de vista técnico, tanto a técnica original (Cox I) como a modificada (Cox III), são operações trabalhosas porém reprodutíveis; tempo longo de CEC foi observado em nossa experiência (média de $156,3^{\prime}$ ), semelhante ao obtido por COX et alii ${ }^{7}\left(184^{\prime}\right)$, o que isoladamente não consideramos como fator para aumento de risco operatório. Nos casos de reoperações, não se observou aumento significativo do tempo de CEC, mas sim no tempo total de cirurgia, em função da dissecção e liberação do coração. A mudança da técnica original para a técnica de Cox III, a nosso ver apresenta algumas vantagens; a não abordagem da região do teto do $A E$ e das proximidades do nó sinusal parece ser fator importante no combate a alguns problemas 
JATENE, M. B.; SOSA, E.; JATENE, F. B.; TARASOUTCHI, F.; MONTEIRO, A. C.; SALERNO, P. R.; SOUZA, L. C. B.; PACHÓN MATEOS, J. C.; JATENE, A. D. - Evolução tardia da operação de Cox para fibrilação atrial em valvopatia mitral. Rev. Bras. Cir. Cardiovasc., $10(1): 18-24,1995$.

observados com a técnica original. Dentre estes fatores pode-se citar a baixa resposta cronotrópica ao teste de atropina e ao exercício, observada com a técnica de Cox I em nossa casuística. COX et alii 9. 11 , além de confirmarem nossos achados, apresentam melhora da resposta cronotrópica com a técnica de Cox III, bem como maior incidência de ritmo sinusal ( $75 \%$ contra $44 \%$ ), quando comparam, respectivamente, as técnicas de Cox I e Cox III. Em 7 pts de nossa casuística, não foi utilizada a crioablação nos anéis mitral, tricúspide e seio coronário, como proposto por Cox em sua técnica original, realizando-se apenas dissecção cuidadosa das respectivas regiões; o resultado obtido foi idêntico aos casos em que se utilizou a crioablação, com controle da arritmia a curto e longo prazo. Em nosso meio, GREGORI et alii ${ }^{17}$ vêm utilizando rotineiramente esta variável técnica, com bons resultados.

Com relação à ocorrência de FA no pós-operatório imediato, cuja incidência em nossa experiência foi de $11,7 \%$, tal fato não deve ser rigorosamente valorizado em uma fase tão inicial de pós-operatório, já que os pts estão sujeitos aos mesmos desequilíbrios hidro-eletrolíticos que qualquer paciente submetido a cirurgia cardíaca. CRESWELL et alii ${ }^{14}$ e $\operatorname{COX}^{5}$ demonstraram que a FA é a arritmia mais freqüentemente encontrada no pós-operatório de qualquer cirurgia cardiaca, chegando a atingir cerca de $4,9 \%$ de toda a população de pts operados, não portadores de FA prévia. Seu controle deve ser feito através de correção de eventuais desequilíbrios ou por medicação específica. Apesar de citada na literatura ${ }^{7}$, a retenção hídrica observada em alguns pts, em função do grande número de incisões atriais, não constituiu complicação freqüente em nossa casuística, sem que seja possível identificar com clareza a ocorrência de tal complicação.

Podemos afirmar que, em função da experiência acumulada até o momento, a operação de Cox tem lugar certo dentro do arsenal terapêutico de combate à FA, devendo ser indicada nos casos de valvopatias mitrais e FA, desde que não haja alguma contra-indicação formal. Não acreditamos que seja prudente indicar a operação de Cox de uma forma preventiva em certas doenças com potencialidade de desenvolvimento de FA, como proposto por BONCHEK et alii ${ }^{1}$, porém admitimos que seu uso deva ser ampliado a todas as situações já comentadas; ressalva deve ser feita no emprego da técnica aos casos de reoperações, em função do aumento observado de morbi-mortalidade. A evolução tardia dos pts operados demonstra a capacidade da operação em controlar a FA na grande maioria dos pts, com manutenção dos resultados no período estudado.

RBCCV $44205-254$

JATENE, M. B.; SOSA, E.; JATENE, F. B.; TARASOUTCHI, F.; MONTEIRO, A. C.; SALERNO,P. R.; SOUZA, L. C. B.; PACHÓN MATEOS, J. C.; JATENE, A. D. - Late follow-up of "Maze" procedure for atrial fibrillation and mitral valve disease. Rev. Bras. Cir. Cardiovasc., 10 (1): 18-24, 1995.

ABSTRACT: The "Maze" procedure for surgical treatment of chronic atrial fibrillation (AF) described by Cox was performed in 34 patients (pts) from July 91 to October $94 ; 26$ were female and the ages ranged from 35 to $68 \mathrm{y}(50.2 y)$. All pts had mitral valve disease (MVD) and chronic AF ( $>1$ year). Thirty one pts had surgical rheumatic MVD (mitral stenosis in 21; mitral double disfunction in 9) and 3 had mixomatous MVD with severe regurgitation. Twenty eight pts were in FC (NYHA) IV and 6 in FC III preoperatively. Left atrium diameter varied from 4.4 to $6.5 \mathrm{~cm}(5.5 \mathrm{~cm})$. In 21 pts the surgical treatment was performed following the original "Maze" technique and in 13 pts with the modifications proposed by Cox. The surgery was completed with 17 mitral conservative procedures and 17 mitral valve replacements (bovine pericardium bioprostheses). Eight pts had left atrial thrombosis. There were $2(5.8 \%)$ immediate deaths (low cardiac output and severe liver disfunction) and 1 patient died in the 48th day with infeccious complications. The first patient required reoperation for bleeding review. Atrial arrhythmias were observed in 6 pts and 14 required temporary pace-maker. Regular "sinus" rhythm was observed in all pts. No other major complications were observed. In 30 pts with more than 6 months of follow up, 27 (90\%) were in regular rhythm (heart rate from 55 to $87 \mathrm{bpm}$ ), with no antiarrhythmic drugs and atrial contraction demonstrated by ECHO. In conclusion, the "Maze" procedure showed effective to treat $A F$ even in pts with big atrium and MVD, with acceptable immediate and late results. Longer observation is required to a definitive conclusion.

DESCRIPTORS: Cox operation, late follow-up. Atrial fibrillation, surgery. 
JATENE, M. B.; SOSA, E.; JATENE, F. B.; TARASOUTCHI, F.; MONTEIRO, A. C.; SALERNO, P. R.; SOUZA, L. C. B.; PACHÓN MATEOS, J. C.; JATENE, A. D. - Evolução tardia da operação de Cox para fibrilação atrial em valvopatia mitral. Rev. Bras. Cir. Cardiovasc., 10 (1): 18-24, 1995.

\section{REFERÊNCIAS BIBLIOGRÁFICAS}

1 BONCHEK, L. I.; BURLINGAME, M. W.; WORLEY, S. J.; VAZALES, B. E.; LUNDY, E. F. - Cox/maze procedure for atrial septal defects with atrial fibrillation: management strategies. Ann. Thorac. Surg., 55: 607-610, 1993.

2 CAMERON, A.; SCHWARTZ, M. J.; KRONMAL, R. A.; KOSINSKI, A. S. - Prevalence and significance of atrial fibrillation in coronary artery disease (CASS Registry). Am. J. Cardiol., 61: 714-747, 1988.

3 COBLER, J. L.; WILLIAMS, M. E.; GREENLAND, P. Thyrotoxicosis in institutionalized elderly patients with atrial fibrillation. Arch. Intern. Med., 144: 1758-1760, 1984.

4 COX, J. L. - Evolving applications of the maze procedure for atrial fibrillation. Ann. Thorac. Surg., 55: 578$580,1993$.

5 COX, J. L. - A perspective of postoperative atrial fibrillation in cardiac operations. Ann. Thorac. Surg., 56: 405-409, 1993.

6 COX, J. L. - The surgical treatment of atrial fibrillation. IV: Surgical technique. J. Thorac. Cardiovasc. Surg., 101: 584-592, 1991.

7 COX, J. L.; BOINEAU, J. P.; SCHUESSLER, R. B.; KATER, K. M.; LAPPAS, D. G. - Five-year experience with the maze procedure for atrial fibrillation. Ann. Thorac. Surg., 56: 814-824, 1993.

8 COX, J. L.; BOINEAU, J. P.; SCHUESSLER, R. B.; KATER, K. M.; LAPPAS, D. G. - From fisherman to fibrillation: an unbroken line of progress. Ann. Thorac. Surg., 58: 1269-1273, 1994.

9 COX, J. L.; BOINEAU, J. P.; SCHUESSLER, R. B.; JAQUISS, R. D. B.; LAPPAS, D. G. - Modification of the maze procedure for atrial flutter and atrial fibrillation. I: Rationale and surgical results. (No prelo. J. Thorac. Cardiovasc. Surg., 1995).

10 COX, J. L.; CANAVAN, T. E.; SCHUESSLER, R. B.; CAIN, M. E.; LINDSAY, B. D.; STONE, C.; SMITH, P. K.; CORR, P. B.; BOINEAU, J. P. - The surgical treatment of atrial fibrillation. II: Intraoperative electrophysiologic mapping and description of the electrophysiologic basis of atrial flutter and atrial fibrillation. J. Thorac. Cardiovasc. Surg., 101: 406426, 1991.

11 COX, J. L.; JAQUISS, R. D. B.; SCHUESSLER, R. B.; BOINEAU, J. P. - Modification of the maze procedure for atrial flutter and atrial fibrillation. II: Surgical technique of the Maze III procedure. (No prelo, J. Thorac. Cardiovasc. Surg. 1995).

12 COX, J. L.; SCHUESSLER, R. B.; BOINEAU, J. P. - The surgical treatment of atrial fibrillation. I: Summary of the current concepts of the mechanism of atrial flutter and atrial fibrillation. J. Thorac. Cardiovasc. Surg., 101: 402-405, 1991.
13 COX, J. L.; SCHUESSLER, R. B.; D'AGOSTINO Jr., H. J.; STONE, C. M.; CHANG, B. C.; CAIN, M. E.; CORR, P. B.; BOINEAU, J. P. - The surgical treatment of atrial fibrillation. III: Development of definitive surgical procedure. J. Thorac. cardiovasc. Surg., 101: $569-583,1991$.

14 CRESWELL, L. L.; SCHUESSLER, R. B.; ROSENBLOOM, M.; COX, J. L.; Hazards of postoperative atrial arrhythmias. Ann. Thorac. Surg., 56: $539-549,1993$.

15 DIAMONTOPOULOS, E. J.; ANTHOPOULOS, L.; NANAS, S.; MALIARAS, G.; CHRISOS, D.; MOULOPOULOS, S. D. - Detection of arrhythmias in a representative sample of the Athens population. Eur. Heart. J., 8 (Supl. D): 17-19, 1987.

16 FISHER, C. M. - Embolism in atrial fibrillation. In: KULBERTUS, H. E.; OLSSON, S. B.; SCHLEPPER, M. (eds.) - Atrial fibrillation. Molndal, Sweden, A.B. Hassle, 1982, p. 192-210.

17 GREGORI Jr, F.; CORDEIRO, C.; COUTO, W.; SILVA S.; AQUINO, W.; NECHAR, A. - The Cox operation without cryoablation for the treatment of chronic atrial fibrillation. (Aceito para publicação em 1994. Ann. Thorac. Surg).

18 GUIRAUDON, G. M.; CAMPBELL, C. S.; JONES, D. L.; McLELLAN, J. L.; McDONALD, J. L. - Combined sino-atrial node atrioventricular node isolation: a surgical alternative to Hiss bundle ablation in patients with atrial fibrillation. Circulation, 72 (Parte 2): 220, 1985 (Resumo).

19 HIROSAWA, K.; SEKIGUCHI, M.; KASANUKI, H. - Natural history of atrial fibrillation. Heart Vessels, 10: (Supl. 2) 14-23, 1987.

JATENE, A. D.; SOSA, E.; TARASOUTCHI, F.; JATENE, M. B.; POMERANTZEFF, P. M. A. - Tratamento cirúrgico da fibrilação atrial. Procedimento do "labirinto": experiência inicial. Rev. Bras. Cir. Cardiovasc., 7: 107-111, 1992.

21 KOSAKAI, Y.; KAWAGUCHI, A. T.; ISOBE, F.; SASAKO, Y.; NAKANO, K.; EISHI, K.; TANAKA, N.; KITO, Y.; KAWASHIMA, Y. - Cox maze procedure for chronic atrial fibrillation associated with mitral valve disease. J. Thorac. Cardiovasc. Surg., 108 : 1049-1055, 1994.

22 MCCARTHY, P. M.; CASTLE, L. W.; MALONEY, J. D.; THOHMAN, R. G.; SIMMONS, T. W.; WHITE, R. D.; KLEIN, A. L.; COSGROVE III, D. M. - Initial experience with the maze procedure for atrial fibrillation. J. Thorac. Cardiovasc. Surg., 105 : 1077 1087, 1993.

23 MARTIN, A.; BENBOW, L. J.; BUTROUS, G. G. S. LEACH, C.; CAMM, A. J. - Five-year follow-up of 101 elderly subjects by means of long-term ambulatory cardiac monitoring. Eur. Heart J., 5: 592-596, 1984. 
JATENE, M. B.; SOSA, E.; JATENE, F. B.; TARASOUTCHI, F.; MONTEIRO, A. C.; SALERNO, P. R.; SOUZA, L. C. B.; PACHÓN MATEOS, J. C.; JATENE, A. D. - Evolução tardia da operação de Cox para fibrilação atrial em valvopatia mitral. Rev. Bras. Cir. Cardiovasc., 10 (1): 18-24, 1995.

24

ONUNDARSON, P. T.; THORGEIRSSON, G.; JONMUNDSSON, E.; SIGFUSSON, N.; HARDARSON, T. - Chronic atrial fibrillation: epidemiologic features and 14 year follow-up: a case control study. Eur. Heart J., 8: 521-527, 1987.

SAVAGE, D. D.; GARRISON, R. J.; CASTELLI, W. P. - Prevalence of submitral (annular) calcium and its correlates in a general population - based sample (the Framingham Study). Am J. Cardiol, 51: 1375$1378,1983$.

SCHEINMAN, M. M. \& EVANS-BELL, T. - Catheter ablation of the atrioventricular junction: a report of the percutaneous mapping and ablation registry. Circulation, 70: 1024-929, 1984.

27 SCHEINMAN, M.; MORADY, F.; HESS, D. S. - Catheter

induced ablation of the atrioventricular junction to control refractory supraventricular arrhythmias. JAMA, 248: 851, 1981.

28

29

\section{TRES}

TRESEDER, A. S.; SASTRY, B. S.; THOMAS, T. P. YATES, M. A.; PATHY, M. S. - Atrial fibrillation and stroke in elderly hospitalized patients. Age Aging, 15: 89-92, 1986.

30 WILLIAMS, J. M.; UNGERLEIDER, R. M.; LOFLAND, G. K.; COX, J. L. - Left atrial isolation: a new technique for the treatment of supraventricular arrhythmias. J. Thorac. Cardiovasc. Surg., 80: 373-380, 1980. 